Saudi Journal of Oral and Dental Research

Abbreviated Key Title: Saudi J Oral Dent Res

ISSN 2518-1300 (Print) |ISSN 2518-1297 (Online)

Scholars Middle East Publishers, Dubai, United Arab Emirates

Journal homepage: https://saudijournals.com

Review Article

\title{
Pain Management in Temporomandibular Disorders: A Review of the Literature
}

\author{
Lydia Nabil Fouad Melek ${ }^{1 *}$, Tara Renton ${ }^{2}$ \\ ${ }^{1}$ BDS, MSc, PhD, Associate Professor of Oral and Maxillofacial Surgery, Department of Oral and Maxillofacial Surgery, Faculty of Dentistry, \\ Alexandria University, Egypt \\ ${ }^{2} \mathrm{PhD}$ MDSc BDS FDS RCS FRACDS (OMS) FHEA, Professor in Oral Surgery, Department of Oral Surgery, King's College London, UK
}

\begin{abstract}
DOI: $10.36348 /$ sjodr.2020.v05i11.005
| Received: 02.11.2020 | Accepted: 16.11.2020 | Published: 23.11.2020
\end{abstract}

*Corresponding author: Lydia Nabil Fouad Melek

\section{Abstract}

Temporomandibular disorders (TMD's) are defined as "A collective term embracing a number of clinical problems that involve the masticatory muscles, temporomandibular joint and associated structures or both". TMD's are considered a major source of non-dental orofacial pain interfering with normal daily activities and affecting the quality of life. Despite huge amount of research in the diagnosis and management of temporomandibular disorders, there is still no certain therapeutic method that proved to be solely effective in pain management resulting from TMD's. This review article contains a collection for the various therapeutic procedures used for management of TMD pain and discusses the most recent evidence on their effectiveness through comprehensive review of Pubmed for relevant publications within the last decade.

Keywords: Temporomandibular disorders, orofacial pain, pain management, quality of life.

Copyright () 2020 The Author(s): This is an open-access article distributed under the terms of the Creative Commons Attribution 4.0 International License (CC BY-NC 4.0) which permits unrestricted use, distribution, and reproduction in any medium for non-commercial use provided the original author and source are credited.

\section{INTRODUCTION}

The temporomandibular joint (TMJ) is a unique diarthrodial joint that is considered as one of the most complex joints in the human body. According to the American academy of orofacial pain, temporomandibular disorders (TMD's) are defined as "A collective term embracing a number of clinical problems that involve the masticatory muscles, temporomandibular joint and associated structures or both [1]". Consistent with the updated diagnostic criteria of temporomandibular disorders (DC/TMD) by Schiffman et al. [2], the most common temporomandibular disorders are arthralgia, myalgia, local myalgia, myofascial pain, myofascial pain with referral, disc displacement disorders, degenerative joint disease, subluxation and headache attributed to TMD.

TMD's are considered a major source of nondental orofacial pain interfering with normal daily activities and affecting the quality of life [3, 4]. Moreover, pain from the TMJ can radiate to the ears, temple, occipital region, shoulders and cervical region [5].

TMD's are also considered the second most common cause of chronic pain after back pain [2]. Their prevalence ranges from 3.7 to $12 \%$ of the population in some studies [6], other authors have reported a TMD prevalence of 10 to $15 \%$ of the population [7].

\section{Causes of pain in TMD's}

TMD's have a multifactorial etiology where the cause of pain and dysfunction in TMD's may be attributed to several factors including genetic predisposition, local and systemic biological factors, in addition to psychological and behavioral factors [8].

Pressure exerted on the highly innervated retro-discal tissues by the condyle in cases of anterior disc displacement may lead to vasoconstriction and release of nitric oxide, reactive oxygen species and thiobarbituric acid, resulting in pain [9, 10]. However, not everyone having disc displacement evident on magnetic resonance images (MRI's) suffers from clinical signs and symptoms of TMD's. This may be due to the high adaptive and remodeling capacity of the TMJ, which explains the minimal or even absent pain in asymptomatic subjects with displaced discs [11].

Pain may also result from different inflammatory conditions affecting the joint including degenerative joint disease, infectious arthritis, 
rheumatic arthritis and metabolic arthritic conditions [12].

Another significant cause of TMD pain is excessive tension of the masticatory muscles [13]. Parafunction and exposure of the joint to microtrauma or macro-trauma may as well, be the predisposing factors to TMD pain.

Association of TMD's with comorbid pain conditions: TMD's may present in association with primary headaches, pain conditions in other parts of the body as chronic back pain or systemic pain conditions as fibromyalgia. This may indicate increased susceptibility of the patient to chronic pain in multiple sites as a result of dysfunction of the pain pathways through peripheral and/or central sensitization [8, 14].

\section{Relation between pain and psychosocial function}

The biopsychosocial model of pain recognizes cognitive, emotional and behavioral aspects of pain in addition to the sensory component, which affect the pain experience and coping strategies. Axis II of DC/TMD includes measures for comprehensive assessment of psychological function in TMD patients. These tools screen the patient for pain intensity, psychosocial distress, and pain-related disability [2].

Several studies have emphasized the psychosocial impact of orofacial pain where the results confirmed that patients with chronic TMD pain exhibit significant psychological dysfunction and impaired health-related quality of life $[15,16]$. This highlights the great importance of TMD pain management not only in relieving the physical suffering but also in improving the psychosocial quality of the patients' lives. Inadequate treatment of TMD pain which lasts for more than 6 months may result in its transformation into chronic pain where peripheral and central sensitization occur as a result of neuroplastic changes contributing to the pain chronicity [17]. A supporting evidence for central sensitization by La Touche et al. [18] is based on finding central and spinal hyperexcitability in TMD patients demonstrated by increase in mechanical temporal summation.

Axis II (DC/TMD) screening instruments are indicated in case of TMD pain lasting for 6 months or more without adequate response to treatment in order to reveal the need for psychological intervention as part of a multidisciplinary approach [2].

\section{Management of pain in TMD's}

Despite huge amount of research in the diagnosis and management of temporomandibular disorders, there is still no certain therapeutic method that proved to be solely effective in pain management resulting from TMD's. According to the Royal College of Surgeons' Primary Care guidance for TMD [19], the main objectives of TMD management are encouragement of self-management strategies through patient education, reducing pain and improvement of function. Therapeutic options mainly include occlusal splints, medications, physiotherapy, behavioral therapies and surgery. The effectiveness of each of those options has been thoroughly investigated in the literature during the last decades. Peripheral, central and/or behavioral positive influences have been suggested as possible mechanisms for the different therapies $[4,20]$.

\section{Selection of the appropriate therapeutic method should take into account the following considerations}

1. Proper diagnosis of the TMD through thorough clinical examination and if indicated, imaging and laboratory investigations.

2. Delivering the treatment within a biopsychosocial context taking into account the biological, psychological and social factors, where psychosocial factors play an important role in response to treatment in case of chronic pain [21].

3. $75-90 \%$ of patients respond well to conservative measures [22].

4. A considerable percentage of TMD patients have comorbid headaches or other pain conditions which may complicate the diagnosis and affect treatment outcome. The inclusion of a neurologist within a multidisciplinary team helps to increase the chances of treatment success in such cases [23].

\section{Diagnosis of TMD's}

All the details of DC/TMD including a symptom questionnaire, a detailed examination protocol and examination form which standardizes the examination and recording of findings in addition to Axis II screening tools are available with the most recent updated versions on the electronic page of the International Network for Orofacial pain and Research Methodology (INFORM) [24].

A few highlights of the DC/ TMD quoted from the INFORM [24] website are provided here as essential recommendations for the clinician during TMD diagnosis:

- Time frame for the DC/TMD is pain present within the last 30 days.

- The examiner asks the patient to point to the location of pain, this indicates the structures affected. According to subsequent clinical examination, the examiner will record the pain location as "joint" or "muscle".

- The examiner asks the patient about current history of headaches.

- Bilateral palpation of the masseter and temporalis muscles is sufficient for diagnostic 
yield in $99 \%$ of the time in case of pain originating from masticatory muscles.

- One finger palpation is recommended with 0.5 to $1.0 \mathrm{~kg}$ pressure for at least 2 seconds or 5 seconds (in case of referred pain) followed by "familiar" pain inquiry and if positive by the "referred" pain inquiry. Goal of the examination is to replicate the patient's pain to locate the source. Each major muscle is divided into 3 zones to ensure that they are examined in a consistent manner.

- Maxillary and mandibular incisors serve as stable landmarks for measurement of the mandibular range of motion. In their absence, the nasopalatine papilla and estimated mandibular midline are used instead.

- A mm ruler starting at the zero mark should be used for measurement of maximal interincisal opening.

- Vertical incisal overlap (positive or negative) is added to the measurement of vertical movement.

- Horizontal overjet (positive or negative) is added to the measurement of protrusive movement.

- The examiner should ask the patient to slowly open 3 times and record any corrected or uncorrected deviation during opening.

- 3 types of open movements (pain-free opening, maximum unassisted opening and maximum assisted opening) are performed during examination.

- Patients should bring posterior teeth into maximal inter-cuspal position before each open-close cycle.

- Joint noises and locking during opening or closing should be noted by the examiner.

Figure 1 (A and B) are diagnostic decision trees which serve as helpful flow charts during TMD diagnosis [available from the INFORM website].

\section{Diagnostic challenge of TMD's}

The complexity of the anatomical structure in the orofacial region and common co-existence of multiple painful conditions can make accurate diagnosis a difficult process [25].

TMD's can mimic toothache, co-exist with headaches and cervicogenic pain or be a part of chronic widespread pain condition [25]. A recent study has also shown that migraine may present (although rare) as isolated facial pain affecting the second or third divisions of the trigeminal nerve [26].

Wrong diagnosis of orofacial pain may lead to ineffective and sometimes irreversible treatments. The holistic approach by using Axis I and Axis II for assessment in addition to appropriate investigations via a multidisciplinary team will help in proper management of patients and protecting them from unnecessary restorative or surgical procedures [25].

Treatment procedures for TMD's can be broadly categorized into non-invasive, minimally invasive and invasive therapies (Figure-2).

Non-invasive treatment options

Patient education and reassurance

Patients are educated about the nature and etiology of their condition and instructed to change some of their habits as avoiding chewing gum, extreme movements or wide opening of the jaw. They are also advised to follow a soft food diet and apply hot/cold fomentations. Following these self-care management strategies might help considerably in decreasing their pain [27]. It is also important for the clinician to emphasize, during patient education, upon the contributory role of psychological factors in pain intensity and persistence [21].

\section{Splint/appliance therapy}

There are many types of appliances that are based on expert opinion rather than high quality research evidence from randomized controlled clinical trials with adequate sample size. The most commonly used splints for TMD management are stabilization and anterior repositioning appliances [28-30]. Splint therapy has been postulated to provide significant reduction of pain in temporomandibular disorders [29]. A metaanalysis by Zhang et al., [30] has revealed that splint therapy helped to reduce the painful episodes in patients suffering from TMJ clicking. Several mechanisms were proposed to explain the action of splint therapy on the joint. The most likely are prevention of tooth contact, breaking parafunctional habits, reduction of muscle tension and unloading the joint.

However, a recent systematic review studying the clinical and cost effectiveness of oral splints has reached the conclusion that there is a very low-quality evidence in the literature to confirm that oral splints effectively reduce pain in TMD's as a group of conditions [31].

Splints may be soft or hard with full coverage of the upper or lower arch. Al-Moraissi et al. [32] have compared the various types of occlusal splints in their network meta-analysis and found that the best results were obtained by hard stabilization splints when combined with counselling therapy.

\section{Jaw Exercises}

Both active and relaxation (static) masticatory muscle exercises performed three or more times daily for ten minutes each day have been proved to effectively reduce TMD pain. Reduction of pain leads consequently to improved range of mandibular motion. Some studies have concluded that relaxation exercises 
have given better results compared to active exercises in alleviation of masticatory muscle tension [13].

\section{Physiotherapy}

Different methods of physiotherapy as massage, ultrasound, short wave diathermy, iontophoresis, laser therapy and transcutaneous electronic nerve stimulation (TENS) have been used throughout the literature and their effect in reducing pain and inflammation has been demonstrated [33, 34].

$\mathrm{Xu}$ et al., [35] in their meta-analysis have concluded the effective role of low-level laser therapy in relief of pain and functional improvement of TMD symptoms. Ultrasound has also given promising results in terms of pain reduction and improving muscular function [27].

When properly administered, physiotherapy provides relief of discomfort and functional improvement at least for a short term and since no harm is usually anticipated, its use is often encouraged as a non-invasive therapy [21].

\section{Pharmacological therapy}

Medications used for TMD pain commonly include analgesics, non-steroidal anti-inflammatory drugs (NSAID's), opioids, anxiolytics, antidepressants, and muscle relaxants.

NSAID's available as over the counter or prescription pain killers are usually effective in case of mild to moderate pain resulting from acute inflammatory joint conditions. To reduce pain effectively, they should be used regularly for at least 2 weeks, taking into consideration their adverse effects especially on the gastrointestinal tract, kidneys and bleeding tendency [36].

Opioids as codeine and oxycodone are used in case of moderate to severe chronic TMD pain. [37]. However, their use is restricted to prescription by welltrained clinicians in selected cases due to the potential of these drugs to cause tolerance and physical dependence in addition to other adverse effects and drug interactions [36].

Muscle relaxants have been commonly prescribed by clinicians to alleviate TMD pain resulting from muscle hyperactivity as in clenching and nocturnal bruxism. A single dose at bed time is usually prescribed. These are centrally acting skeletal muscle relaxants that should be used with caution as they induce increased sedation [36].

Antidepressants in low doses whether tricyclic antidepressants or selective serotonin reuptake inhibitors (SSRI's) have been used as neuro-modulatory agents in patients with refractory TMD pain. Many patients with TMD have comorbid depression and sleep disturbances which may be managed by these medications, contributing to the indirect improvement of TMD pain symptoms. However, consultation with the patient's physician is necessary for management of potential side effects $[36,38]$.

Benzodiazepines have been used as anxiolytics and anticonvulsants. They also act as muscle relaxants and sleep inducers. Although some studies have proved the effectiveness of these agents in reducing TMD pain [39], their use is discouraged due to the numerous side effects, tendency to induce tolerance and physical dependence with prolonged use [37, 40].

\section{Psychological management including cognitive behavioral therapy (CBT)}

Pain is stressful and living with chronic pain implies stress that may lead to anxiety, depressed mood and reduced quality of life. Stress also plays an important role in amplification and persistence of pain. This confirms the importance of adjunctive psychological management in patients with chronic TMD's [21].

Cognitive behavioral therapy is based on changing the patterns of thinking or behavior that are behind people's difficulties and so changing the way they feel. There is available evidence on the added benefit of CBT in effective pain relief when combined with usual treatment for patients with persistent TMD's [41]. CBT has been proven to provide long term improvement of TMD symptoms in terms of pain severity, depression and activity interference [42].

\section{Acupuncture}

This method is well known in Asian countries and started to become popular in the western world. Some studies have pointed to the positive effect of acupuncture in reducing pain of TMD especially in patients with myofascial pain symptoms [43].

\section{Minimally invasive treatment options Arthrocentesis}

Joint lavage using two needles was first introduced by Nitzan et al., [44] as a minimally invasive and efficient technique (especially for TMJ internal derangement) that can be performed under local anesthesia for release of adhesions, wash out of inflammatory mediators, improving limitation of mouth opening and pain relief. Studies using the classic double puncture technique or the alternative single puncture technique have stated marked reduction of pain following arthrocentesis, owing to the effective flush out of pain mediators, pressure relief and decrease of joint loading [10, 45, 46]. Patients with acute pain seem to respond better to arthrocentesis than those with chronic pain [47] A review has reported $83.5 \%$ success rate when arthrocentesis was performed for patients with internal derangement (disc displacement without reduction) and osteoarthritis [45]. 


\section{Intra-articular injections}

Throughout the literature, different medications and substances have been injected into the joint. In most instances, they were used as adjunctive to arthrocentesis.

The most common medications used after arthrocentesis are corticosteroids, sodium hyaluronate and non-steroidal anti-inflammatory drugs (NSAID's). Some studies have proved an added benefit of pain management when intra-articular injections are performed in adjunct to arthrocentesis while other studies have shown that no significant benefit has been drawn from those additional injections in comparison to arthrocentesis alone [10, 48-52].

Other studies have investigated the injection of corticosteroids with local anesthesia into the joint and reported good results in terms of pain management and improvement of function [53]. The corticosteroids commonly used intra-articular are betamethasone, methylprednisolone, dexamethasone and triamcinolone [54]. Corticosteroids suppress the inflammatory process inside the joint via prevention of the expression of cytokines and inflammatory mediators through binding to specific receptors [51]. In spite of the reported high benefit of injectable corticosteroids in management of TMD pain, they still have serious side effects as hypertension, damage of the fibrous layer and bone resorption especially with repeated injections [55].

Hyaluronic acid (HA) represents the main component of synovial fluid responsible for lubrication and nutrition of tissues inside the TMJ, so its intraarticular injection has been found to provide long term improvement of TMD symptoms [56]. There is also evidence that application of HA helps to regulate the inflammatory mediators in osteoarthritis of the TMJ [57].

Prolotherapy involving the injection of $25 \%$ dextrose into the joint has given promising results in terms of pain reduction and improvement of other TMD symptoms when used in patients with TMJ internal derangement [58]. Prolotherapy has also been used with success in case of joint hypermobility where pericapsular injection of dextrose helped to strengthen the capsule and ligaments leading to efficient management of recurrent joint dislocation [59].

Effective reduction of TMD pain has also been obtained in studies applying intra-articular injection of platelet-rich plasma (PRP) $[60,61]$ where the analgesic effect of PRP was suggested to be related to the upregulation of cannabinoid receptors $\mathrm{CB} 1$ and $\mathrm{CB} 2$ [62].

\section{Botulinum toxin type a injection}

Machado et al., [63] in their recent systematic review have shown that injection of Botulinum toxin type A for painful TMJ disorders was well tolerated and was more effective in pain reduction compared to placebo and other treatment modalities. Patel et al., [64] have recommended considering the use of Botulinum toxin for TMD's only after exhaustion of other conservative measures.

A recent randomized study has compared the use of Botulinum toxin alone, stabilization splint alone and both together for management of bruxism. The pain scores had decreased significantly in the Botulinum toxin group and the combination group in comparison to the splint group [65].

\section{Arthroscopy}

This technique involves the insertion of an arthroscope into the joint under general anesthesia for diagnostic or therapeutic purposes. It can be used to perform lysis and lavage under direct visualization in addition to other operative joint procedures.

In the meta-analysis by Rigon et al., [66], arthroscopy has resulted in better improvement of maximal interincisal opening after 12 months compared to arthrocentesis. However, no significant difference was found between arthrocentesis and arthroscopy in terms of pain reduction.

\section{Invasive Treatment Options}

Patients not responding to non-invasive and minimally invasive therapies may be candidates for more invasive surgical treatments including arthroplasty, discectomy, disc repositioning, disc plication and other open joint surgeries reaching to total joint replacement. Total replacement of the joint in case of severe degeneration of the articular surfaces damaged by arthritis, has resulted in satisfactory improvement of symptoms and function [67]. However, open TMJ surgeries may carry the risk of complications including wound infection, facial nerve injury and fatal vascular injuries [56].

Open joint surgery was found to be superior to arthroscopic surgery in patients with TMJ internal derangement in terms of pain reduction with comparable results in other clinical parameters [68]. 


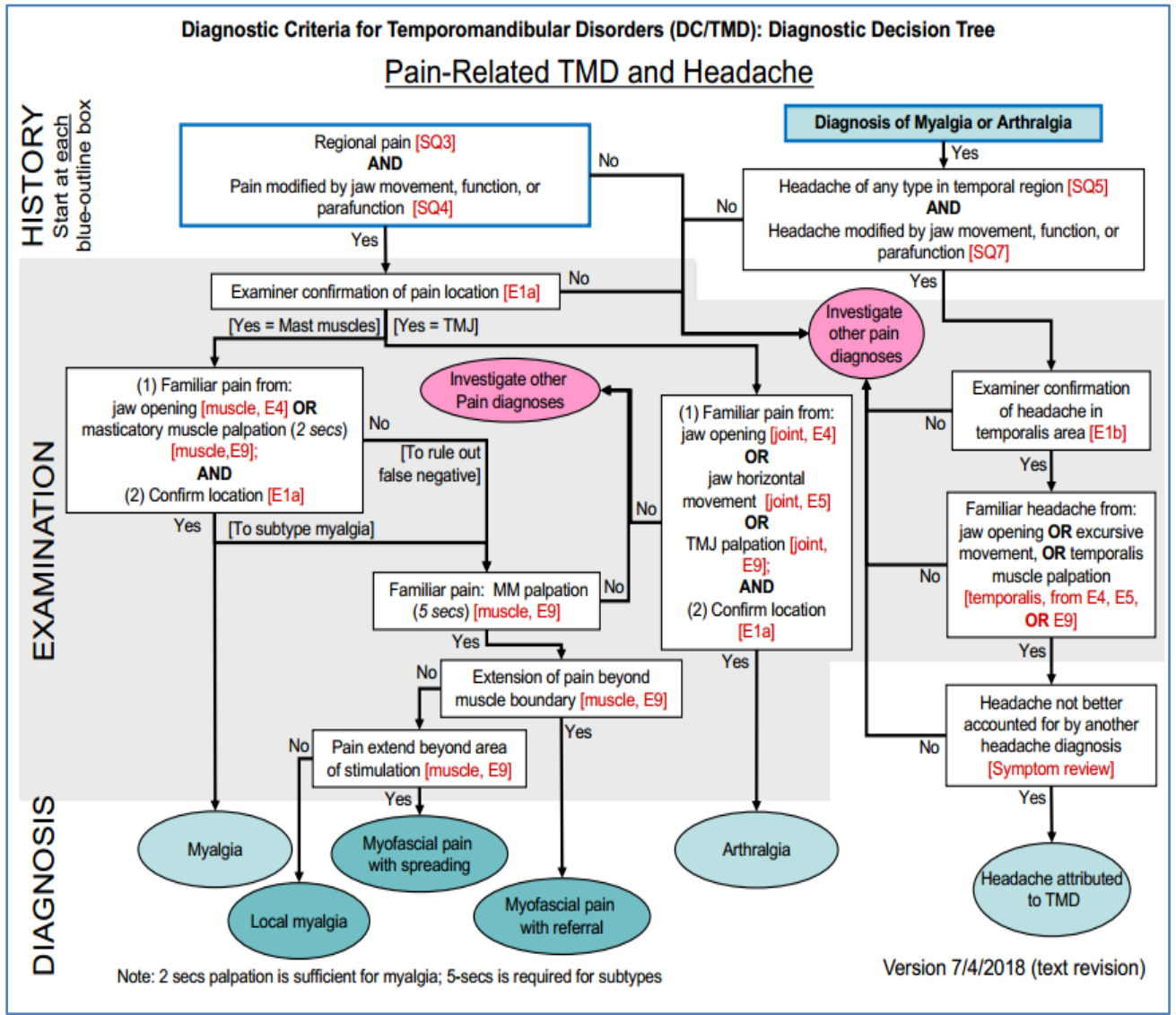

Fig-1A: Diagnostic decision tree (available from https://buffalo.app.box.com/s/btzm3fzeocw5xmh3ty2x5gjtba23hai5, accessed on 20 August 2020)

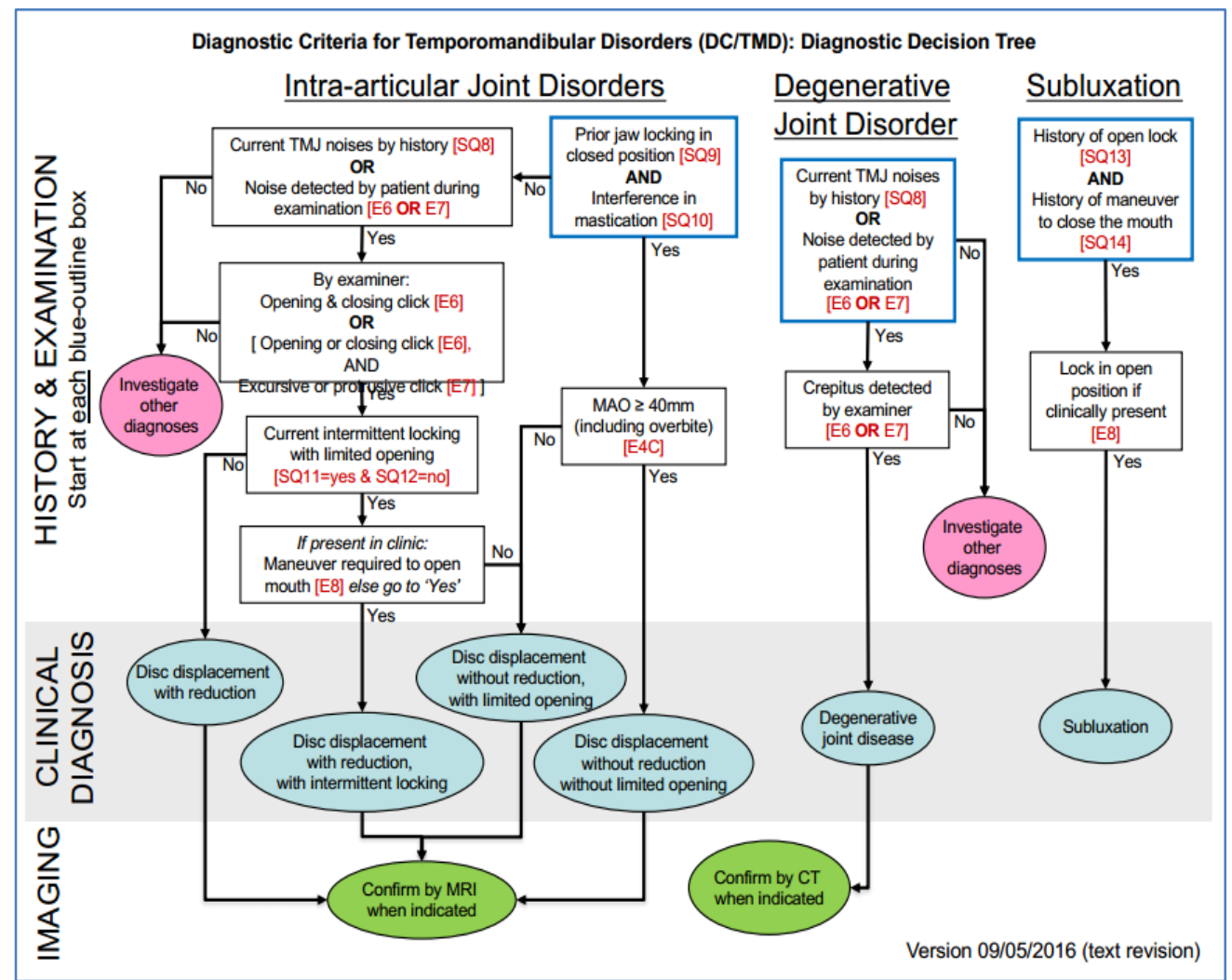

Fig-1B: Diagnostic decision tree (available from https://buffalo.app.box.com/s/btzm3fzeocw5xmh3ty2x5gjtba23hai5, accessed on 20 August 2020) 


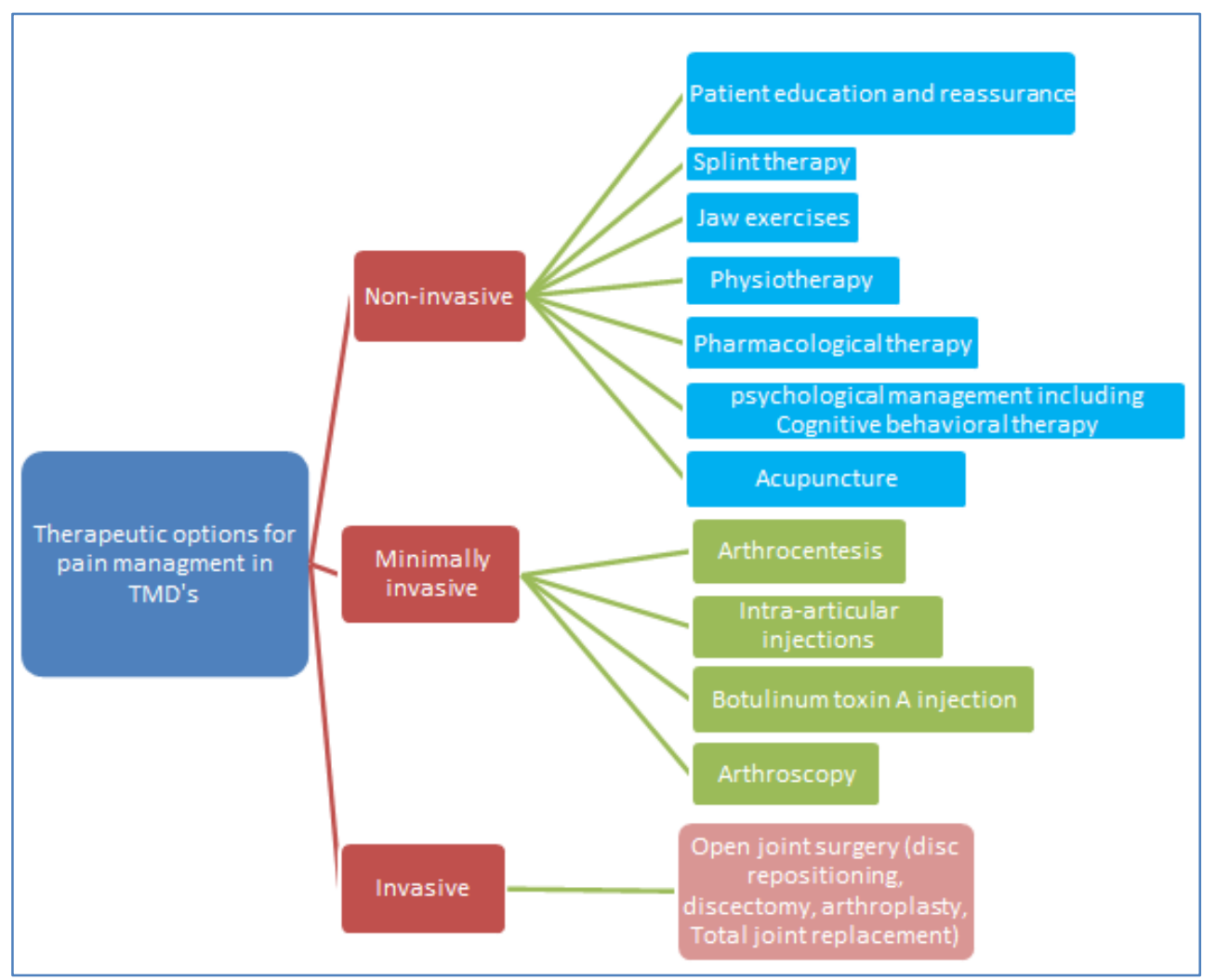

Fig-2: Diagram showing the categories of pain management options in TMD's

\section{A paradigm shift in the hierarchy of TMD treatment}

It was traditionally accepted among clinicians that all conservative non-invasive measures should be first applied for painful TMD's before shifting to the next level of minimally invasive procedures. However, there is a recently emerging evidence that minimally invasive procedures especially intra-articular injections of pharmacologic agents with or without arthrocentesis is more effective in pain reduction and improvement of maximal mouth opening than conservative measures both in the short ( $\leq 6$ months) and intermediate term ( 6 months- 4years) levels. This advocates the benefit for the early use of minimally invasive therapies as soon as the patient does not show initial response to conservative treatment [68].

\section{REFERENCES}

1. Okeson, J. (2013). Functional anatomy and biomechanics of masticatory system In: Proceedings of the Management of Temporomandibular Disorders and Occlusion, 120.

2. Schiffman, E., Ohrbach, R., Truelove, E., Look, J., Anderson, G., Goulet, J. P., ... \& Michelotti, A. (2014). International RDC/TMD Consortium Network, International association for Dental Research; Orofacial Pain Special Interest Group, International Association for the Study of Pain. $J$ Oral Facial Pain Headache, 28(1), 6-27.

3. Armijo-Olivo, S., Pitance, L., Singh, V., Neto, F., Thie, N., \& Michelotti, A. (2016). Effectiveness of manual therapy and therapeutic exercise for temporomandibular disorders: systematic review and meta-analysis. Physical therapy, 96(1), 9-25.

4. Conti, P. C. R., Pinto-Fiamengui, L. M. S., Cunha, C. O., \& Conti, A. C. D. C. F. (2012). Orofacial pain and temporomandibular disorders: the impact on oral health and quality of life. Brazilian oral research, 26(SPE1), 120-123.

5. Walczynska-Dragon, K., \& Baron, S. (2011). The biomechanical and functional relationship between temporomandibular dysfunction and cervical spine pain. Acta of Bioengineering and Biomechanics, 13(4), 93-98.

6. Magnusson, T., Egermark, I., \& Carlsson, G. E. (2000). A longitudinal epidemiologic study of signs and symptoms of temporomandibular disorders from 15 to 35 years of age. Journal of orofacial pain, 14(4).

7. Ohrbach, R., Fillingim, R. B., Mulkey, F., Gonzalez, Y., Gordon, S., Gremillion, H., ... \& Maixner, W. (2011). Clinical findings and pain symptoms as potential risk factors for chronic TMD: descriptive data and empirically identified domains from the OPPERA case-control study. The Journal of Pain, 12(11), T27-T45.

8. Maixner, W., Diatchenko, L., Dubner, R., Fillingim, R. B., Greenspan, J. D., Knott, C., \& Slade, G. D. (2011). Orofacial pain prospective evaluation and risk assessment study-the OPPERA study. The journal of Pain, 12(11), T4T11.

9. Shaefer, J. R., Riley, C. J., Caruso, P., \& Keith, D. (2012). Analysis of criteria for MRI diagnosis of 
Lydia Nabil Fouad Melek \& Tara Renton., Saudi J Oral Dent Res, Nov, 2020; 5(11): 552-561

$\begin{array}{lrr}\text { TMJ disc } & \text { displacement } & \text { and } \\ \text { arthralgia. International } & \text { Journal } & \text { of }\end{array}$ Dentistry, 2012.

10. Alpaslan, C., Bilgihan, A., Alpaslan, G. H., Güner, B., Yis, M. Ö., \& Erbaş, D. (2000). Effect of arthrocentesis and sodium hyaluronate injection on nitrite, nitrate, and thiobarbituric acid-reactive substance levels in the synovial fluid. Oral Surgery, Oral Medicine, Oral Pathology, Oral Radiology, and Endodontology, 89(6), 686-690.

11. Kumar, R., Pallagatti, S., Sheikh, S., Mittal, A., Gupta, D., \& Gupta, S. (2015). Suppl 2: M4: Correlation Between Clinical Findings of Temporomandibular Disorders and MRI Characteristics of Disc Displacement. The open dentistry journal, 9, 273.

12. Ahmad, M., \& Schiffman, E. L. (2016). Temporomandibular joint disorders and orofacial pain. Dental Clinics, 60(1), 105-124.

13. Bae, Y., \& Park, Y. (2013). The effect of relaxation exercises for the masticator muscles on temporomandibular joint dysfunction (TMD). Journal of physical therapy science, 25(5), 583-586.

14. Bonato, L. L., Quinelato, V., De Felipe Cordeiro, P. C., De Sousa, E. B., Tesch, R., \& Casado, P. L. (2017). Association between temporomandibular disorders and pain in other regions of the body. Journal of oral rehabilitation, 44(1), 9-15.

15. Smith, J. G., Karamat, A., Melek, L. N., Jayakumar, S., \& Renton, T. (2020). The differential impact of neuropathic, musculoskeletal and neurovascular orofacial pain on psychosocial function. Journal of Oral Pathology \& Medicine, 49(6), 538-546.

16. Gustin, S.M., Wilcox, S.L., Peck, C.C., Murray, G.M., Henderson, L.A. (2011). Similarity of suffering: equivalence of psychological and psychosocial factors in neuropathic and nonneuropathic orofacial pain patients. Pain, 152: 825-832.

17. Renton, T. (2019). ChroniC Pain and overview or differential diagnoses of non-odontogeniC orofaCial Pain. Primary Dental Journal, 7(4), 7186.

18. La Touche, R., Paris-Alemany, A., Hidalgo-Pérez, A., López-de-Uralde-Villanueva, I., Angulo-DiazParreño, S., Muñoz-García, D. (2018). Evidence for Central Sensitization in Patients with Temporomandibular Disorders: A Systematic Review and Meta-analysis of Observational Studies. Pain Pract; 18: 388-409.

19. La Touche, R., Paris- Alemany, A., HidalgoPérez, A., López- de- Uralde- Villanueva, I., Angulo- Diaz- Parreño, S., \& Muñoz- García, D. (2018). Evidence for central sensitization in patients with temporomandibular disorders: a systematic review and meta- analysis of observational studies. Pain Practice, 18(3), 388409.
20. Conti, P.C., de Alencar, E.N., da Mota, Corrêa, A.S., Lauris, J.R., Porporatti, A.L., Costa, Y.M. (2012). Behavioural changes and occlusal splints are effective in the management of masticatory myofascial pain: a short-term evaluation. J Oral Rehabil, 39: 754-760.

21. Beecroft, E., Penlington, C., Desai, H., \& Durham, J. (2019). Temporomandibular Disorder for the General Dental Practitioner. Primary dental journal, 7(4), 62-70.

22. Greene, C. S. (2001). The etiology of temporomandibular disorders: implications for treatment. Journal of orofacial pain, 15(2), 93105.

23. Karamat, A., Smith, J. G., Lambru, G., \& Renton, T. (2019). Changing face of orofacial pain: The diagnostic impact of working with Neurology on an orofacial pain clinic. International journal of oral and maxillofacial surgery, 48(7), 924-929.

24. International Network for Orofacial pain and Research Methodology. https://ubwp.buffalo.edu/rdc-tmdinternational/.

25. Renton, T. (2020). Tooth-Related Pain or Not? Headache, 60: 235-246.

26. Lambru, G., Elias, L. A., Yakkaphan, P., \& Renton, T. (2020). Migraine presenting as isolated facial pain: A prospective clinical analysis of 58 cases. Cephalalgia, 0333102420933277.

27. Wieckiewicz, M., Boening, K., Wiland, P., Shiau, Y. Y., \& Paradowska-Stolarz, A. (2015). Reported concepts for the treatment modalities and pain management of temporomandibular disorders. The journal of headache and pain, 16(1), 106.

28. Fricton, J., Look, J. O., Wright, E., Alencar Jr, F. G., Chen, H., Lang, M., \& Miriam Velly, A. (2010). Systematic review and meta-analysis of randomized controlled trials evaluating intraoral orthopedic appliances for temporomandibular disorders. Journal of orofacial pain, 24(3), 237.

29. Ebrahim, S., Montoya, L., Busse, J. W., CarrascoLabra, A., Guyatt, G. H., \& Medically Unexplained Syndromes Research Group. (2012). The effectiveness of splint therapy in patients with temporomandibular disorders: a systematic review and meta-analysis. The Journal of the American Dental Association, 143(8), 847-857.

30. Zhang, C., Wu, J. Y., Deng, D. L., He, B. Y., Tao, Y., Niu, Y. M., \& Deng, M. H. (2016). Efficacy of splint therapy for the management of temporomandibular disorders: a metaanalysis. Oncotarget, 7(51), 84043.

31. Riley, P., Glenny, A. M., Worthington, H. V., Jacobsen, E., Robertson, C., Durham, J., ... \& Boyers, D. (2020). Oral splints for patients with temporomandibular disorders or bruxism: a systematic review and economic evaluation. Health Technology Assessment (Winchester, England), 24(7), 1.

32. Al-Moraissi, E. A., Farea, R., Qasem, K. A., AlWadeai, M. S., Al-Sabahi, M. E., \& Al-Iryani, G. 
M. (2020). Effectiveness of occlusal splint therapy in the management of temporomandibular disorders: network meta-analysis of randomized controlled trials. International Journal of Oral and Maxillofacial Surgery.

33. De Laat, A., Stappaerts, K., \& Papy, S. (2003). Counseling and physical therapy as treatment for myofascial pain of the masticatory system. Journal of orofacial pain, 17(1).

34. de Souza, R. F., Da Silva, C. H. L., Nasser, M., Fedorowicz, Z., \& Al- Muharraqi, M. A. (2012). Interventions for managing temporomandibular joint osteoarthritis. Cochrane database of systematic reviews, (4).

35. Xu, G. Z., Jia, J., Jin, L., Li, J. H., Wang, Z. Y., \& Cao, D. Y. (2018). Low-level laser therapy for temporomandibular disorders: a systematic review with meta-analysis. Pain Research and Management, 2018.

36. Ouanounou, A., Goldberg, M., \& Haas, D. A. (2017). Pharmacotherapy in Temporomandibular disorders: A review. J Can Dent Assoc, 83(h7), 1488-2159.

37. Haas, D. A. (1995). Pharmacologic considerations in the management of temporomandibular disorders. Journal (Canadian Dental Association), 61(2), 105.

38. Lynch, M. E. (2001). Antidepressants as analgesics: a review of randomized controlled trials. Journal of Psychiatry and Neuroscience, 26(1), 30.

39. Harkins, S., Linford, J., Cohen, J., Kramer, T., \& Cueva, L. (1991). Administration of clonazepam in the treatment of TMD and associated myofascial pain: a double-blind pilot study. Journal of Craniomandibular Disorders, 5(3).

40. Guaiana, G., \& Barbui, C. (2016). Discontinuing benzodiazepines: best practices. Epidemiology and Psychiatric Sciences, 25(3), 214-216.

41. Randhawa, K., Bohay, R., Côté, P., van der Velde, G., Sutton, D., Wong, J. J., ... \& Stupar, M. (2016). The effectiveness of noninvasive interventions for temporomandibular disorders. The Clinical journal of pain, 32(3), 260-278.

42. Aggarwal, V. R., Lovell, K., Peters, S., Javidi, H., Joughin, A., \& Goldthorpe, J. (2011). Psychosocial interventions for the management of chronic orofacial pain. Cochrane Database of Systematic Reviews, (11).

43. Wu, J. Y., Zhang, C., Xu, Y. P., Yu, Y. Y., Peng, L., Leng, W. D., ... \& Deng, M. H. (2017). Acupuncture therapy in the management of the clinical outcomes for temporomandibular disorders: a PRISMA-compliant metaanalysis. Medicine, 96(9).

44. Nitzan, D. W., Dolwick, M. F., \& Martinez, G. A. (1991). Temporomandibular joint arthrocentesis: a simplified treatment for severe, limited mouth opening. Journal of Oral and Maxillofacial Surgery, 49(11), 1163-1167.

45. Monje-Gil, F., Nitzan, D., \& González-Garcia, R. (2012). Temporomandibular joint arthrocentesis. Review of the literature. Medicina oral, patologia oral y cirugia bucal, 17(4), e575.

46. Singh, S., \& Varghese, D. (2013). Single puncture arthrocentesis of temporomandibular joint; introducing a novel device: A pilot study. National Journal of Maxillofacial Surgery, 4(2), 193.

47. Emshoff, R., \& Rudisch, A. (2004). Determining predictor variables for treatment outcomes of arthrocentesis and hydraulic distention of the temporomandibular joint. Journal of oral and maxillofacial surgery, 62(7), 816-823.

48. Ferreira, N., Masterson, D., de Lima, R. L., de Souza Moura, B., Oliveira, A. T., da Silva Fidalgo, T. K., ... \& Grossmann, E. (2018). Efficacy of viscosupplementation with hyaluronic acid in temporomandibular disorders: A systematic review. Journal of Cranio-Maxillofacial Surgery, 46(11), 1943-1952.

49. Clavero, M. A. G., Sanz, M. V. S., Til, A. M., \& Palacio, J. B. (2019). Prospective study to evaluate the influence of joint washing and the use of hyaluronic acid on 111 arthrocentesis. Oral and Maxillofacial Surgery, 23(4), 415-421.

50. Antonarakis, G. S., Courvoisier, D. S., Hanquinet, S., Dhouib, A., Carlomagno, R., Hofer, M., \& Scolozzi, P. (2018). Benefit of temporomandibular joint lavage with intra-articular steroids versus lavage alone in the Management of Temporomandibular Joint Involvement in juvenile idiopathic arthritis. Journal of Oral and Maxillofacial Surgery, 76(6), 1200-1206.

51. Kiliç, S. C. (2016). Does injection of corticosteroid after arthrocentesis improve outcomes of temporomandibular joint osteoarthritis? A randomized clinical trial. Journal of Oral and Maxillofacial Surgery, 74(11), 21512158.

52. AL-Said, S. N., Shawky, N., \& Ragab, H. R. (2015). Comparative study of arthrocentesis with or without using piroxicam in the management of temporomandibular joint disorders. Alexandria Dental Journal, 40(2), 160-165.

53. Samiee, A., Sabzerou, D., Edalatpajouh, F., Clark, G. T., \& Ram, S. (2011). Temporomandibular joint injection with corticosteroid and local anesthetic for limited mouth opening. Journal of oral science, 53(3), 321-325.

54. Davoudi, A., Khaki, H., Mohammadi, I., Daneshmand, M., Tamizifar, A., Bigdelou, M., \& Ansaripoor, F. (2018). Is arthrocentesis of temporomandibular joint with corticosteroids beneficial? A systematic review. Medicina oral, patologia oral y cirugia bucal, 23(3), e367.

55. Haddad, I. K. (2000). Temporomandibular joint osteoarthrosis. Saudi Med J, 21, 675-679. 
56. De Rossi, S. S., Greenberg, M. S., Liu, F., \& Steinkeler, A. (2014). Temporomandibular disorders: evaluation and management. Medical Clinics, 98(6), 1353-1384.

57. Iturriaga, V., Bornhardt, T., Manterola, C., \& Brebi, P. (2017). Effect of hyaluronic acid on the regulation of inflammatory mediators in osteoarthritis of the temporomandibular joint: a systematic review. International Journal of Oral and Maxillofacial Surgery, 46(5), 590-595.

58. Hassanien, N., Kamel, H. M., \& Rashed, S. A. (2020). Dextrose Prolotherapy versus Low Level Laser Therapy (LLLT) for Management of Temporomandibular Joint Disorders (TMD): Clinical Randomized controlled Study. Egyptian Dental Journal, 66(1-January (Oral Surgery)), 95106.

59. Kiliç, S. C., \& Güngörmüş, M. (2016). Is dextrose prolotherapy superior to placebo for the treatment of temporomandibular joint hypermobility? A randomized clinical trial. International journal of oral and maxillofacial surgery, 45(7), 813-819.

60. Gupta, S., Sharma, A. K., Purohit, J., Goyal, R., Malviya, Y., \& Jain, S. (2018). Comparison between intra-articular platelet-rich plasma injection versus hydrocortisone with local anesthetic injections in temporomandibular disorders: A double-blind study. National Journal of Maxillofacial Surgery, 9(2), 205.

61. Bousnaki, M., Bakopoulou, A., \& Koidis, P. (2018). Platelet-rich plasma for the therapeutic management of temporomandibular joint disorders: a systematic review. International journal of oral and maxillofacial surgery, 47(2), 188-198.

62. Lee, H. R., Park, K. M., Joung, Y. K., Park, K. D., \& Do, S. H. (2012). Platelet-rich plasma loaded hydrogel scaffold enhances chondrogenic differentiation and maturation with up-regulation of CB1 and CB2. Journal of Controlled Release, 159(3), 332-337.

63. Machado, D., Martimbianco, A. L. C., Bussadori, S. K., Pacheco, R. L., Riera, R., \& Santos, E. M. (2019). Botulinum toxin type A for painful temporomandibular disorders: Systematic review and meta-analysis. The Journal of Pain.

64. Patel, J., Cardoso, J. A., \& Mehta, S. (2019). A systematic review of botulinum toxin in the management of patients with temporomandibular disorders and bruxism. British dental journal, 226(9), 667-672.

65. Yurttutan, M. E., Sancak, K. T., \& Tüzüner, A. M. (2019). Which treatment is effective for bruxism: Occlusal splints or botulinum toxin?. Journal of Oral and Maxillofacial Surgery,77(12), 24312438.

66. Rigon, M., Pereira, L. M., Bortoluzzi, M. C., Loguercio, A. D., Ramos, A. L., \& Cardoso, J. R. (2011). Arthroscopy for temporomandibular disorders. Cochrane Database of Systematic Reviews, (5).

67. Tanaka, E., Detamore, M. S., \& Mercuri, L. G. (2008). Degenerative disorders of the temporomandibular joint: etiology, diagnosis, and treatment. Journal of dental research, 87(4), 296307.

68. Al-Moraissi, E. A. (2015). Open versus arthroscopic surgery for the management of internal derangement of the temporomandibular joint: a meta-analysis of the literature. International Journal of Oral and Maxillofacial Surgery, 44(6), 763-770. 